\title{
L'approccio del counseling nutrizionale al paziente affetto da obesità
}

\author{
Oliveri Emanuela1, Scaramelli Fabio², Macagno Stefania ${ }^{3}$ \\ ${ }^{1}$ Dietista, Counselor presso Officina di Counseling Nutrizionale Bra, Italy \\ ${ }^{2}$ Counselor sistemico presso Officina di Counseling Nutrizionale Bra, Italy \\ ${ }^{3}$ Dietista con competenze di Counseling presso Officina di Counseling Nutrizionale Bra, Italy
}

\section{Parole chiave}

Counseling Nutrizionale

Dietoterapia

Obesità

Relazione

Resistenza al cambiamento

Trattamento

\begin{abstract}
Riassunto
Nella dietoterapia, il soggetto obeso rappresenta un paziente ostico, con già alle spalle, spesso, diversi percorsi nutrizionali; non di rado la sua percezione di autoefficacia risulta già intaccata, oltre che dai diversi fallimenti e recuperi ponderali, anche dallo stigma sociale (non di rado presente anche in ambiente sanitario) nei confronti di questa patologia: un quadro di difficile gestione per il professionista della nutrizione. II Counseling Nutrizionale consiste in un insieme di tecniche di conduzione dell'incontro nutrizionale volte alla costruzione di un buon clima relazionale; esso offre un aiuto, proponendo un atteggiamento non giudicante: il soggetto è il protagonista della terapia, che viene co-costruita su misura, sulla base delle informazioni che il paziente, unico esperto della propria vita, fornisce. L'esplorazione spinta dalla curiosità, l'ascolto attivo, il lavoro sulle risorse, l'aumento della percezione di autoefficacia del paziente, il focus sul come, il colloquio motivazionale, sono solo alcune delle tecniche che permettono di creare una relazione terapeutica che agevola il crollo delle barriere di resistenza del paziente. In questo modo, è possibile spostare il punto di vista sugli aspetti che davvero ostacolano il raggiungimento dell'obiettivo, lavorarci e, di conseguenza, aiutare il soggetto ad applicare risorse e strategie utili al processo del cambiamento.
\end{abstract}

\begin{abstract}
The obese subject represents a tricky patient for the nutrition professionals, often having several nutritional interventions behind him already; his feeling of self-efficacy has frequently been undermined already, not only from the several failures and weight re-gains, but also from the social stigma (not uncommon to be found in the healthcare setting, too) towards this disease: a picture difficult to manage for the nutrition professional. The Nutritional Counseling consists of a set of techniques of conducting the nutritional meeting, aimed at creating a good relational climate; it comes to help, through a non-judgemental approach: the individual is the protagonist of the therapy, which is co-constructed and tailored, on the basis of the information which the patient, who is the unique expert on his life, provides. The exploration out of curiosity, the active listening, the work on the resources, the increase of the patient's feeling of selfefficacy, the focus on how, the motivational interviewing are just a few of the techniques which allow to create a therapeutic relationship which facilitates the fall of the patient's resistance barriers. In this way, it is possible to move the point of view on the aspects which really hinder the goal achievement, to work on them and, consequently, to help the individual implement the resources and strategies useful for the changing process.
\end{abstract}

Copyright ( 2020 Oliveri Emanuela et al. This is an open-access article distributed under the terms of the Creative Commons Attribution License (CC BY). The use, distribution or reproduction in other forums is permitted, provided the original author(s) and the copyright owner(s) are credited and that the original publication in this journal is cited, in accordance with accepted academic practice. No use, distribution or reproduction is permitted which does not comply with these terms.

Oliveri Emanuela (凶) info@officinacounselingnutrizionale.it

Received: 20 April 2020; Accepted: 27 April 2020; Published online: 30 April 2020. doi:10.32044/ijedo.2020.03 


\section{Introduzione}

La dietoterapia incontra uno scoglio importante nella gestione del paziente affetto da obesità, la pratica clinica presenta infatti elevati livelli di drop-out: secondo una revisione sistematica di 121 studi comprendenti interventi di dimagrimento, il drop-out corrisponde al 37\% dei partecipanti in un anno di follow-up (Elobeid et al, 2009). Secondo lo studio QUOVADIS, alla base di tali numeri sembrerebbero intervenire numerosi fattori, quali aspettative troppo elevate rispetto al calo ponderale, motivazioni alla perdita di peso che coinvolgono principalmente l'aspetto estetico, la percezione dei progressi effettuati come non soddisfacenti; tuttavia, nel numero di drop-out sono considerati anche coloro che hanno abbandonato il percorso in quanto soddisfatti dei risultati raggiunti o con una percezione di auto-efficacia tale da proseguire con il dimagrimento senza aiuti esterni (Dalle Grave et al, 2014). Inoltre, anche di coloro che riescono a raggiungere il loro obiettivo ponderale, una buona parte è destinata a recuperare interamente il peso perso: i dati di letteratura parlano di un ritorno al peso di partenza nell'arco di 3-5 anni (Greaves et al, 2017). Ne consegue che i dati epidemiologici relativi alla prevalenza dell'obesità non sono rosei, al punto che l'OMS non stenta a definirla una vera e propria epidemia globale: sovrappeso e obesità interessano il $30-50 \%$ degli adulti e fino a un terzo dei bambini delle regioni europee (Branca et al, 2007). In Italia le stime non sono molto diverse, infatti la prevalenza negli adulti con eccesso di peso corrisponde al $45,9 \%$, mentre il $10,5 \%$ si colloca nella fascia dell’obesità (Bologna, 2018).

\section{Limiti della dietoterapia prescrittiva}

Della popolazione adulta con problemi di sovrappeso od obesità, corrispondente a quasi la metà dell'intera popolazione italiana, è rilevante la quantità che intraprende un percorso di dimagrimento, il quale può presentarsi come un percorso ostico, che richiede un vero e proprio stravolgimento delle abitudini, cambiamenti comportamentali da applicare con costanza nella propria quotidianità. Chi si trova a lottare con il proprio peso conosce molto bene le difficoltà che si possono incontrare nel cambiare in modo permanente il proprio stile di vita, che, volendole semplificare, si possono racchiudere in due aree distinte: quella del metodo con cui si cerca il cambiamento di peso e quella del mantenimento dei risultati.
Nonostante sia ormai risaputo che un dimagrimento troppo rapido non è raccomandabile in un'ottica di salute (Freedman et al, 2001; Neumark-Sztainer et al, 2006; Cairella et al, 2017), sono ancora molte le persone che tentano soluzioni empiriche per rimettersi in forma velocemente per poi scontrarsi con la poca fattibilità o salubrità delle stesse. Alcuni tentativi sono così poco sostenibili e distanti da un'alimentazione equilibrata da essere abbandonati ancor prima che i risultati ponderali inizino a essere evidenti, con l'ovvio ritorno alle abitudini precedenti. La difficoltà del dimagrimento emerge anche nel caso in cui ci si rivolga non al fai da te, bensì a professionisti della nutrizione: pur trattandosi di dietoterapie tecnicamente ineccepibili, non di rado accade che il percorso venga interrotto proprio nel momento in cui si è giunti al termine del dimagrimento e ci si dovrebbe approcciare alla fase più importante, quella del mantenimento e così, come dimostrano i dati, la nuova forma fisica, faticosamente raggiunta, non viene mantenuta a lungo. Accade spesso, dunque, che la persona con obesità si ritrovi ad accumulare più di un'esperienza nel complesso fallimentare presso professionisti della nutrizione, nel tentativo di trovare finalmente la soluzione giusta per sé. Tenendo in considerazione la letteratura, ciò che ne consegue è il famoso effetto yo-yo (Brownell e Rodin, 1994; Marchesini et al, 2004; Mackie et al, 2017; Cairella et al, 2017). Una delle plausibili conseguenze di questa sequenza di fatti è la diminuzione della percezione personale di autoefficacia e della fiducia nelle figure sanitarie che si occupano di nutrizione.

Questo è lo scenario che si può trovare il professionista quando incontra un paziente affetto da obesità, ossia il confronto con un bagaglio di esperienze dietoterapiche che esso porta con sé corredate da informazioni teoriche affidabili che, tuttavia, in qualche modo non hanno funzionato. Il rischio di rappresentare, per quella persona, l'ennesimo sanitario con il quale fare l'ennesimo tentativo non riuscito è altissimo, con il possibile esito di confermare la sfiducia verso la categoria dei sanitari, cui è più facile attribuire le precedenti débacle, e di indebolire ulteriormente il senso di autoefficacia del paziente (Byrne, 2002; Marchesini et al, 2004; Texeira et al, 2015).

Come ci dicono i numeri, questa tipologia di paziente si presenta con grande frequenza al professionista della nutrizione, presentando per le ragioni sopracitate una notevole difficoltà al trattamento. In ragione delle tante e diverse complicanze metaboliche e patologie associate all'obesità, il professionista sanitario si trova ad affrontare anche un carico di responsabilità nei confronti del costo sociale che potrebbe derivare da un fallimento della dietoterapia. 


\section{Stigma sociale del peso}

Sull'autoefficacia della persona che si ha davanti agisce in maniera negativa la pressione sociale per la quale l'obesità è una condizione che, ai nostri giorni, è ancora oggetto di stigmatizzazione. Lo stigma sociale del peso è definito come una serie di "comportamenti e convinzioni negativi diretti verso gli individui per il loro peso e solitamente si manifesta attraverso stereotipi (es: chi è in sovrappeso è pigro), pregiudizi negli atteggiamenti (es: atteggiamenti negativi da parte da parte dei datori di lavoro) e comportamenti discriminatori (es: ignorare gli individui sovrappeso). Si verifica in diversi ambiti (es: ambiente educativo e sanitario), da diverse fonti (es: amici e gli stessi individui affetti da obesità)" (Puhl e Heuer, 2010; Papadopoulos e Brennan, 2015). Lo stigma nasce non soltanto dalla sempre maggiore importanza dell'estetica nella società attuale, ma soprattutto dalla convinzione che, a differenza delle altre patologie, l'obesità sia una condizione della quale il soggetto è responsabile in prima persona: ecco che spuntano, quindi, giudizi riferiti alle presunte caratteristiche personali, quali la pigrizia, la mancanza di motivazione o di autodisciplina. In sintesi, la persona è vista come incapace di limitare ciò che mangia, ed è da questa interpretazione che ne consegue la sua colpevolizzazione (Jung et al, 2015). Non di rado, inoltre, accade che il soggetto con obesità si senta vittima di pregiudizi e stereotipi all'interno dello stesso ambiente sanitario da cui dovrebbe ricevere aiuto (Jung et al, 2015). È quindi possibile incontrare un paziente che ha, oltre a un bagaglio di esperienze sfavorevoli, anche una lunga esperienza di stigmatizzazione sociale della propria condizione, stigma al quale rischia di aderire lui stesso facendone la propria storia dominante (Quadrino, 2009) dando vita a ciò che Watzlawick ha definito come "profezia che si autodetermina" (Watzlawick et al, 1971), fenomeno che può essere implicato in reazioni capaci di abbattere, ulteriormente, il suo senso di autoefficacia (Bandura, 2000).

\section{Counseling nutrizionale}

È in questa cornice complessa che può rendersi utile uno strumento come il counseling nutrizionale, che prevede l'implementazione delle competenze tecniche nutrizionali con le tecniche di comunicazione consapevole ed efficace, che permettono la costruzione di un buon clima relazionale con il paziente, considerandolo nella sua unicità e come unico e solo esperto della propria vita: il professionista con competenze di counseling nutrizionale entra nel mondo dell'altro con la cautela necessaria a formare e mantenere una relazione buona abbastanza da essere funzionale allo scopo terapeutico (Oliveri e Scaramelli, 2019), con un atteggiamento non giudicante e con la curiosità di avere un nuovo mondo da esplorare.

Il counseling nutrizionale prevede diverse predisposizioni mentali basilari: una di queste è la curiosità. Lo scopo del professionista è di aiutare il paziente a raggiungere i suoi obiettivi di salute: per farlo, questi due mondi, quello del paziente e quello del sanitario, devono entrare in una relazione buona abbastanza da essere terapeutica. Ciò è possibile soltanto tramite l'esplorazione operata dal professionista, spinta dalla curiosità rivolta alla storia del paziente, scevra da giudizi e interpretazioni, che si rende esplicita invitando il paziente, attraverso le giuste domande, a esprimere le sue esperienze passate, le sue conoscenze, i suoi timori e dubbi, le sue sensazioni riguardo alla sua condizione attuale; una curiosità che apre anche alle immagini e sensazioni che potrebbe avere in un fisico diverso. Questo è possibile soltanto se si assume di non sapere nulla della persona che si ha di fronte: le domande e le riflessioni che devono guidare il professionista sono: "Che cosa so di questa persona? Che cosa mi serve sapere? È l'unica esperta della propria vita e soltanto lei mi può orientare al suo interno per trovare insieme una via percorribile". Diventa importante, dunque, confrontarsi il meglio possibile con i propri pregiudizi, che potrebbero creare dei filtri impedendo al professionista di esplorare tutte le strade possibili e portarlo a ricondurre quel particolare paziente a stereotipi riguardanti pazienti con storie simili, ma non uguali, alla sua: a far correre questi rischi sono le scorciatoie mentali che noi tutti utilizziamo nella vita comune (Quadrino, 2009).

Le tecniche di counseling nutrizionale hanno come base l'ascolto attivo, una modalità relazionale in cui chi ascolta concentra completamente la propria attenzione sulla storia dell'interlocutore, per aiutarlo ad esprimersi e carpire il più possibile di quello che ci sta dicendo: è un "ascolto perché voglio capire" (Quadrino, 2009). È diverso da un ascolto passivo, in cui chi ascolta si limita ad ascoltare e basta: il professionista interviene per porre domande, fare riassunti e commenti, per riordinare la narrazione e per assicurarsi di aver colto correttamente ciò che il paziente intende dire e, infine, per agevolarlo nella prosecuzione del suo racconto. Il paziente diventa il protagonista della terapia nutrizionale, e in ciò si differenzia da metodologie meno attente alla narrazione, prescrittive e con una comunicazione a senso unico, dove il professionista è colui che dà istruzioni e il paziente colui il quale le riceve, con il compito di metterle in atto passivamente. Il counseling nutrizionale, al con- 
trario, prevede un pieno coinvolgimento del paziente, non soltanto nella stesura del piano alimentare, che deve tener conto delle sue abitudini e dei suoi gusti, ma soprattutto nelle modalità e nelle strategie che possono permettergli di stare nello schema. Se egli si trova di nuovo davanti a un professionista della nutrizione significa che qualcosa nelle sue esperienze passate non ha funzionato: come già accennato, egli ha già tutte le istruzioni da applicare per far avvenire il dimagrimento. Evidentemente manca un passaggio che gli permetta di metterle in atto o, meglio, di attuarle in modo costante. Questo è un altro compito del counseling nutrizionale: il professionista con tecniche di counseling nutrizionale ha gli strumenti per spostare il punto di vista: occorre, infatti, concentrarsi sul come seguire le indicazio$\mathrm{ni}$, piuttosto che sul che cosa mangiare. Nuovamente, entra in gioco il ruolo da protagonista del paziente: le azioni da mettere in atto partono proprio dalle sue caratteristiche individuali. Il professionista si serve delle domande per far emergere dal racconto del paziente tutte quelle risorse che possono tornare utili nel percorso di dimagrimento con il fine di personalizzarlo per il massimo possibile sulle attitudini della singola persona. In questo modo cambia anche il concetto di responsabilizzazione che tanto torna nella stigmatizzazione del peso: il paziente viene sì responsabilizzato, ma non come colpevole della propria patologia, bensì in quanto fulcro e protagonista della terapia dietetica.

Con le tecniche del counseling nutrizionale si costruisce una relazione di fiducia tra paziente e professionista, funzionale al passaggio delle informazioni necessarie e alla costruzione della partnership necessaria in questo tipo di terapia. Gli incontri di counseling nutrizionale rappresentano degli spazi in cui il paziente si può sentire a suo agio abbastanza da svelarsi al professionista, per condividere con lui le difficoltà che sta affrontando. In questo spazio, si fanno cadere le barriere di resistenza del paziente, permettendo al professionista di andare oltre e vedere che cosa gli impedisca di mettere in pratica la teoria, portando alla luce quei pensieri che il paziente ha su se stesso che possono costituire delle vere e proprie trappole, pensieri limitanti che contribuiscono a negare l'accesso al cambiamento di stile di vita (Nardone, 2013).

Si introduce, così, il concetto di ambivalenza: in ogni processo di cambiamento, ci sono sempre due forze che spingono in senso contrario. Da un lato vi è, appunto, la spinta al cambiamento, alimentata, nel nostro caso, dalle motivazioni al dimagrimento; d'altro canto, invece, vi è la resistenza al cambiamento, sempre presente, di minore o maggiore entità. (Milner e Rollnick, 2004). Per un paziente affetto da obesità questa resistenza al cambiamento può consistere, ad esempio, nella libertà di mangiare ciò che vuole o nell'impegno che dovrà porre nell'organizzazione dei suoi pasti; oppure, si può trattare di elementi che hanno radici più profonde, quali l'utilizzo del proprio fisico come strumento di difesa o di distanziamento rispetto al mondo fuori di lui; o ancora, la fisicità come simbolo di imponenza e autorità. Nel caso di questi ultimi esempi, che hanno radici profonde, il professionista con tecniche di counseling nutrizionale non possiede i mezzi necessari per gestire questi pensieri: egli deve effettuare l'invio a un'altra figura, che possa scavare più a fondo, là dove il counseling nutrizionale non può e non ha il mandato di arrivare. Il lavoro che viene effettuato sulla persona, dunque, è di tipo multidisciplinare: il counseling nutrizionale permette di far venire a galla gli ostacoli, che vanno affrontati, non soltanto per il raggiungimento dei risultati, ma soprattutto per superare quei pensieri limitanti che, nel lungo termine, svolgono il loro effetto impedendo il raggiungimento di un equilibrio stabile. Per ottenere un intervento il più possibile efficace e omogeneo, sarebbe auspicabile che tutte le figure coinvolte (non soltanto, quindi, anche il medico di base e altri specialisti che hanno in cura il paziente, come l'endocrinologo, il diabetologo, ecc) facessero uso delle tecniche di counseling.

Nell'incontro di counseling nutrizionale una volta fatta emergere la "spinta opposta" (Selvini Palazzoli et al, 1975) al cambiamento, il professionista potrà lavorarci, esplorando quanto forte sia questa spinta e quanto rischia di inficiare il percorso iniziato insieme al paziente. Tramite l'esplorazione, il sanitario con tecniche di counseling nutrizionale ha precedentemente raccolto informazioni preziose che possono tornare utili in questa fase: egli avrà individuato determinate risorse del paziente, le sue aspettative, gli ostacoli e i suoi pensieri limitanti che lo bloccano. Inoltre, sarà a conoscenza degli obiettivi del paziente ed è in particolare su questi ultimi che agirà nell'effettuazione del colloquio motivazionale. Il professionista cerca di comprendere, sempre attraverso le domande, quanto siano importanti tali obiettivi per il paziente e quanto, invece, sia forte la spinta al mantenimento dello status quo. Egli domanda al paziente, con una metafora, di metterle sui due piatti della bilancia, per capire quale abbia il maggior peso al momento. Nel caso in cui gli obiettivi siano più importanti, li si può utilizzare enfatizzandoli per controbilanciare le attenzioni che il paziente dovrà porre nelle sue scelte alimentari e il lavoro che egli dovrà fare per contrastare la spinta di resistenza al cambiamento. In caso contrario, significa che il paziente si trova ancora in uno stadio di contemplazione e non è ancora pronto all'azione: agire in questo momento sarebbe precoce e, con molte probabilità, porterebbe un fallimen- 
to. In questi casi, occorre fermarsi e lavorare ancora sulla motivazione del paziente, con l'obiettivo di farlo riflettere e aiutarlo a spostarsi da una posizione all'altra della ruota del cambiamento (Prochaska et al, 1994). Meglio, in questi casi, partire dopo per arrivare prima (Nardone, 2009).

\section{Conclusioni}

Un limite del presente studio consiste nella mancanza di dati a supporto dell'efficacia del counseling nutrizionale rispetto alla dietoterapia tradizionale. Sono necessari, di conseguenza, studi clinici controllati per confrontare l'efficacia dell'intervento di counseling nutrizionale rispetto al trattamento dietetico prescrittivo tradizionale.

In conclusione, sono diversi gli strumenti, dei quali si è citata solo una piccola parte, che il counseling nutrizionale offre per la gestione del paziente con obesità. Attraverso tali tecniche il professionista considera il paziente come unico, permettendogli di osservare la sua situazione da un altro punto di vista e di munirsi dell'equipaggiamento adatto ad affrontare il viaggio. Per stare nella metafora, il counseling nutrizionale permette al professionista e al paziente di affrontare la scalata di una vetta con l'equipaggiamento necessario a tale impresa e non con lo zainetto adatto ad una scampagnata. Si tratta di un percorso che richiederà tempo ed energia a entrambi, ma di cui il paziente continuerà a raccogliere i frutti per molto tempo.

\section{Bibliografia}

Bandura, A. (2000). "Autoefficacia”. Trento: ed. Erikson.

Bologna, E. (2018). Sovrappeso e obesità. In "Fumo, alcol, alimentazione, eccesso ponderale e prevenzione" Capitolo del Rapporto Osservasalute 2018, 44-45.

Branca, F., Nikogosian, H., Lobstein, T. (2007). La sfida dell'obesità nella Regione europea dell'OMS e le strategie di risposta. Compendio. Geneva: WHO. Traduzione italiana curata dal Ministero della Salute e dalla Società Italiana di Nutrizione Umana.

Brownell, K.D., Rodin, J. (1994). Medical, Metabolic, and Psycholocal Effects of Weight Cycling. Archives of Internal Medicine, 154: 1325-1330.

Byrne, S.M. (2002). Psychological aspects of weight maintenance and relapse in obesity. Journal of Psychosomatic Research, 53:1029-1036.

Cairella, G., De Virilis, C., Donini, L.M., Ghiselli, A., Giampietro, M., Migliaccio, P.A., Sette, S., Scognamiglio, U., Ticca, M. Centro di ricerca Alimenti e Nutrizione. (2017). Attenti alle diete e agli integratori. Linee guida per una sana alimentazione, dossier scientifico, edizione 2017, 11: 1241-1253.
Dalle Grave, R., Calugi, S., Marchesini, G. (2014). The influence of cognitive factors in the treatment of obesity from the QUOVADIS study. Behaviour Research and Therapy, 63: 157-161.

Elobeid, M.A., Padilla, M.A. et al. (2009). Missing Data in Randomized Clinical Trials for Weight Loss: Scope of the Problem, State of the Field, and Performance of Statistical Methods. PLoS One, 4(8): e 6624.

Freedman, M.R., King, J., Kennedy, E. (2001). Popular diets: a scientific review. Obesity Research, 9(1), 1S-40S.

Greaves, C., Poltawski, L., Garside, R., Briscoe, S. (2017). Understanding the challenge of weight loss maintenance: A systematic review and synthesis of qualitative research on weight loss maintenance. Health Psychology Review, 11(2):145-163.

Jung, F.U.C.E., Luck-Sikorski, C., Wiemers, N., Riedel-Heller, S.G. (2015). Dietitians and Nutritionists: Stigma in the Context of Obesity. A Systematic Review. PLoS One, 10(10): e0140276. doi:10.1371/journal.pone.0140276

Mackie, M.G., Samocha-Bonet, D., Tam, C.S. (2017) Does weight cycling promote obesity and metabolic risk factors? Obesity Research \& Clinical Practice, 11(2):131-139.

Marchesini, G., Cuzzolaro, M., Mannucci, E., Dalle Grave, R., Gennaro, M., Tomasi, F., Barantani, E.G., Melchionda, N and the QUOVADIS Study Group. (2004). Weight cycling in treatment-seeking obese persons: data from the QUOVADIS study. International Journal of Obesity, 28: 1456-1462.

Milner, W.R., Rollnick, S. (2004). Il colloquio motivazionale. Trento: ed. Erikson.

Nardone, G. (2009). Problem solving Strategico da tasca. Milano: Adriano Salani Editore.

Nardone, G. (2013). Psicotrappole. Milano: Adriano Salani Editore.

Neumark-Sztainer, D., Wall, M., Guo, J., Story, M., Haines, J., Eisenberg, M. (2006). Obesity, Disordered Eating, and Eating Disorders in a Longitudinal study of adolescents: how dieters fare 5 years later? Journal of the American Dietetic Association; 106: 559-568.

Oliveri, E., Scaramelli, F. (2019). Piccolo manuale di counseling nutrizionale. Venezia: ed. Onda d'Urto.

Papadopoulos, S., Brennan, L. (2015). Correlates of Weight Stigma in Adults with Overweight and Obesity: a Systematic Literature Review. Obesity, 23 (9): 1743-1760.

Prochaska, J.O., Norcross, J.C., DiClemente, C.C. (1994). Changing for Good. New York City: William Morrow Paperbacks Press.

Puhl, R.M., Heuer, C.A. (2010). Obesity Stigma: Important Considerations for Public Health. American Journal of Public Health, 100 (6): 1019-1028.

Quadrino, S. (2009). Il colloquio di counselling. Torino: Istituto Change.

Selvini Palazzoli, M., Boscolo, L., Cecchin, G., Prata, G. (1975). Paradosso e controparadosso, Milano: ed. Raffaello Cortina Editore.

Texeira, P.J., Carraça, E.V., Marques, M.M., et al. (2015). Successful behavior change in obesity interventions in adults: a systematic review of self-regulation mediators. BioMed Central Medicine, 13:84.

Watzlawick, P., Beavin, J.H., Jackson, D.D. (1971). Pragmatica della comunicazione umana. Roma: ed. Astrolabio. 